\title{
Insights into Vortex Shedding with Data Analysis*
}

\author{
Jiun-Jih MIAU**, J. K. TU*** and T. W. LIAO*** \\ **Department of Aeronautics and Astronautics, National Cheng Kung University, \\ Tainan, Taiwan 70101, ROC \\ E-mail:jjmiau@mail.ncku.edu.tw \\ ***Department of Aeronautics and Astronautics, National Cheng Kung University, \\ Tainan, Taiwan 70101, ROC
}

\begin{abstract}
Unsteady, three-dimensional characteristics of vortex shedding were studied with self-made MEMS sensors situated spanwisely on a circular cylinder subjected to uniform incoming flow. Firstly, verification on the reliability of the MEMS sensor signals was made by comparing with the hot-wire signals obtained in the flow simultaneously. Subsequently, the MEMS sensor signals were analyzed with Wavelet and Hilbert transformations and 2D-FFT. By Wavelet and Hilbert transformations, the results obtained indicate that the strongly three-dimensional vortex shedding events are featured with pronounced spanwise variations in the instantaneous phases of vortex shedding, which are further identified as the occurrences of vortex dislocation. Furthermore, the results of 2D-FFT analysis reveal that the spanwise wave numbers of vortex shedding largely falls in a range between -2 to 2, for the MEMS sensors spanned over a spanwise region of $3 \mathrm{D}$ in length.
\end{abstract}

Key words: Vortex Shedding; MEMS Sensors; Wavelet and Hilbert-Huang Transformations

\section{Introduction}

Three-dimensional characteristics of wake flows have attracted a significant amount of research works in past decades. Roshko ${ }^{1}$ reported that at a Reynolds number of approximately 180, the two-dimensional periodic Strouhal vortex wake undergoes a transition to a three-dimensional appearance. According to Roshko ${ }^{1}$, the three-dimensionality was featured with irregularities in the velocity fluctuations measured in the wake. In a later work, Roshko ${ }^{2}$ attributed the three-dimensionality of vortex shedding to extrinsic and intrinsic effects. Extrinsic effect is mainly associated with geometric configurations of bluff bodies. ${ }^{3-5}$ Intrinsic effect is attributed to the three-dimensional flow instabilities. ${ }^{6-7}$

As pointed out by a number of the investigators, ${ }^{2,8-11}$ the three-dimensional wake flows are featured with dislocations of spanwise shedding vortices. In the visualization of wake flow behind a thin plate for Reynolds number at 200, Eisenlohr and Eckelmann ${ }^{12}$ noticed this phenomenon, termed as vortices splitting. Williamson ${ }^{8}$ showed a visualization photo depicting the natural formation of vortex dislocations in wake flow behind a circular cylinder for Reynolds number at 210 . While this phenomenon is deemed to play an important role in turbulent wake development for Reynolds numbers above $10^{2}$, most of the visualization results reported in the literature were limited to low Reynolds numbers of $10^{2}$. This is understandable, since either dye or smoke-wire visualization technique employed at low Reynolds numbers become less applicable for flows at higher Reynolds numbers.

This work was interested in the flow characteristics of vortex dislocation at Reynolds numbers of $10^{4}$, which was made possible by applying a spanwise array of MEMS (Micro

*Received 5 Dec., 2008 (No. 08-0876) [DOl: 10.1299/jfst.4.415] 
Electrical-and-Mechanical Systems) sensors on a circular cylinder subjected to a uniform incoming flow. This approach has the advantage in obtaining flow information at multiple-points on the surface of the cylinder simultaneously, thus the characteristics of vortex dislocation could be studied and described in a quantitative manner.

At the beginning of this study, the reliability of the MEMS sensor signals was investigated by comparing with the hot-wire signals obtained in the flow. Subsequently, analysis of the MEMS sensors signals was made with Wavelet and Hilbert-Huang transformations to unveil the instantaneous flow behaviors, which were highlighted by the events of vortex dislocation, of which pronounced spanwise phase difference of vortex shedding were noticed. Furthermore, 2D Fast Fourier transformation (2D-FFT) of the signals of the MEMS sensors provided the information concerning the dominant spanwise wave numbers of the three-dimensional vortex shedding structures.

\section{The Flow Characteristics of Interest}

The unsteady, three-dimensional characteristics of vortex shedding concerned in the present study can be elaborated with the results reported in the previous studies, ${ }^{13,14}$ which are briefly described below. A recent work by the present authors (JJM and JKT) ${ }^{13}$ studied the unsteady characteristics of flow separation from a circular cylinder with flow visualization and MEMS thermal film sensors on the surface. As evidenced by the dye flow visualization results obtained in a water channel at Reynolds number of $1.8 \times 10^{4}$, the phenomenon of unsteady flow separation appeared to be strongly three-dimensional. This can be realized in Fig. 1, in which a time series of flow visualization photos reported in Miau et $a l .{ }^{13}$ are reproduced. Tu et $a l .{ }^{14}$ further highlighted the three-dimensional behavior of flow separation over the entire span of a circular cylinder with the flow visualization photos obtained at $\mathrm{Re}=2.31 \times 10^{4}$. These photos are reproduced in Fig. 2 . As seen, some of the dye streaks were tilted toward either spanwise direction, indicated by the arrow signs in the figure, implying the presence of non-zero spanwise velocity near the surface of the cylinder. Moreover, in these photos the locations of flow separation appeared in wavy patterns along the spanwise direction, evidencing the three-dimensionality of flow separation from a circular cylinder.

The present study was aimed to gain better understanding on the instantaneous, three-dimensional characteristics of the flow around a circular cylinder with the application of multiple MEMS sensors reported in the previous works. ${ }^{13,14}$ As will be seen below, this goal was successfully accomplished with the assistance of the data analysis techniques employed.

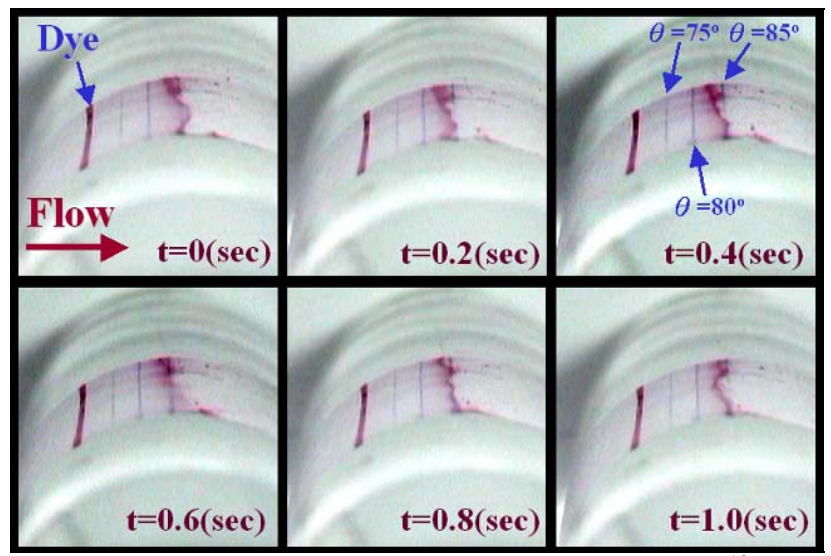

Figure 1 Flow visualization photos reproduced from Miau et al. ${ }^{13}$ of unsteady flow separation on the surface of a circular cylinder at $\mathrm{Re}=1.8 \times 10^{4}$. 


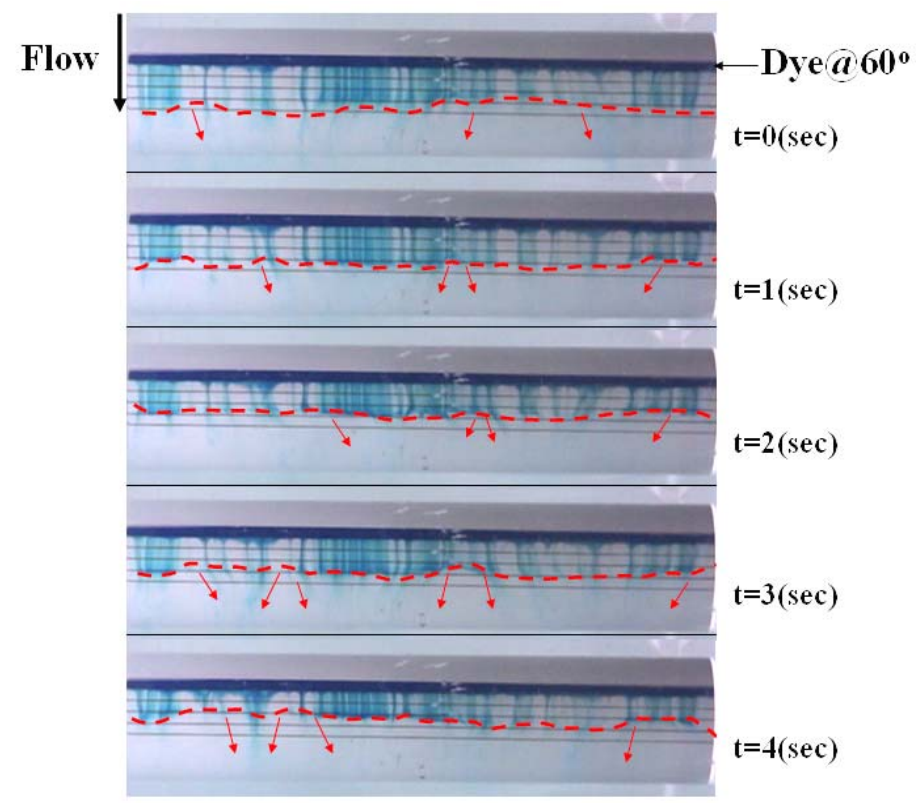

Figure 2 Flow visualization photos reproduced from Tu et al. ${ }^{14}$ of unsteady flow separation over the span of a circular cylinder obtained at $\mathrm{Re}=2.31 \times 10^{4}$.

\section{Experimental Methods}

For this study, experiments were carried out in a closed-return low-speed wind tunnel and an open-type low-speed wind tunnel, respectively. The test section of the former wind tunnel is $150 \mathrm{~mm} \times 160 \mathrm{~mm} \times 1500 \mathrm{~mm}$ in dimension, and the test section of the latter is circular in shape, $500 \mathrm{~mm}$ in diameter and $800 \mathrm{~mm}$ in length.

Shown in Fig. 3 are the schematic drawings depicting the circular cylinder models situated in the test sections of the two wind tunnels. $\theta=0$ denotes the angular position of the forward stagnation point; $x, y$ and $z$ denote the axes along the streamwise, vertical and spanwise directions, respectively, where the origin is located at the center of the circular cylinder.

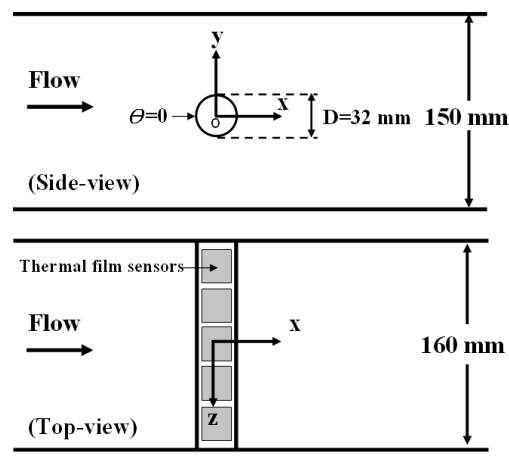

(a)

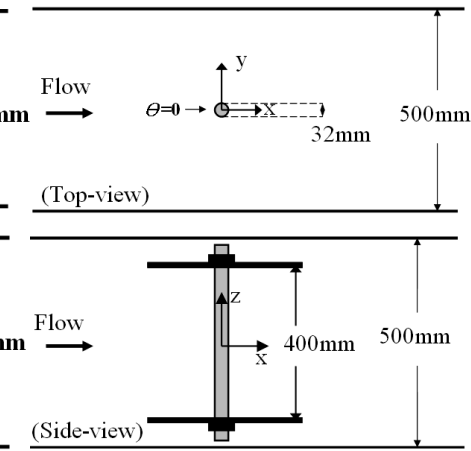

(b)

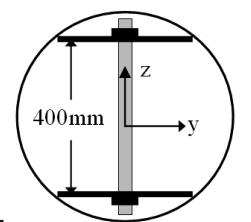

Figure 3 Experimental arrangements of the circular cylinder models in (a) the closed-return low-speed wind tunnel and (b) the open-type low-speed wind tunnel.

Note that in this study the self-made MEMS thermal film sensors were flushed with the surface of the circular cylinder models. Figure 4 (a) gives an example of a circular cylinder model together with the MEMS sensors situated in the test section of the closed-return wind tunnel shown in Fig. 3 (a). The MEMS sensors were made of platinum thin film, $0.1 \mu \mathrm{m}$ in thickness, deposited on a polyimide flexible skin of $20 \mu \mathrm{m}$ in thickness. ${ }^{15}$ As illustrated in Fig. 4 (b), the MEMS sensors fabricated were equally spaced, $1.6 \mathrm{~mm}$ apart, and 14 of which were selected for the present purpose of measurement. 
Since the thickness of the MEMS sensors together with the flexible skin is rather small compared to the diameter of the cylinder, the disturbance caused by the presence of the sensors is assumed negligible in this study.

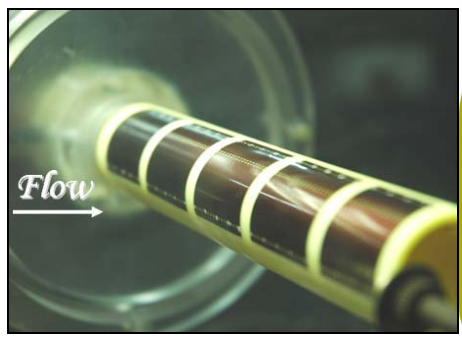

(a)

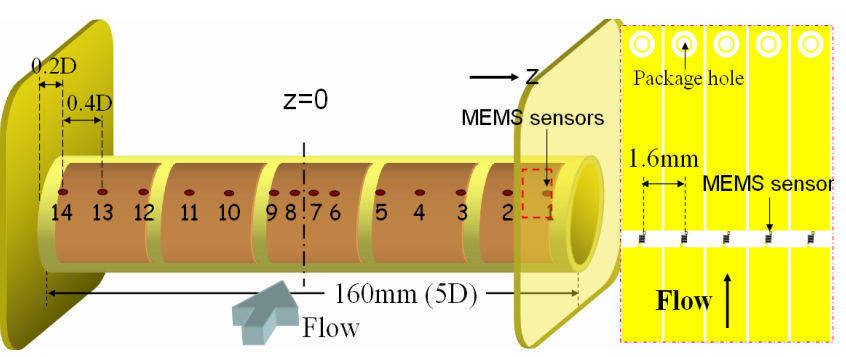

(b)

Figure 4 (a) A photo of the circular cylinder with MEMS sensors in the test section of the closed-return wind tunnel, and (b) schematic drawing of the cylinder model employed with the MEMS sensors aligned spanwisely.

During the experiment, the signals of each MEMS sensor were processed with an AC amplifier circuit. Therefore, the output signals contained no DC component. The signals of multiple MEMS sensors were sampled simultaneously by a data acquisition system featuring 16 channels sample-and-hold capability. The sampling rate chosen was $10 \mathrm{KHz}$ per channel.

In this study, the Reynolds number denoted as Re is defined based on D, the diameter of the cylinder, and $\mathrm{U}_{0}$, the incoming freestream velocity. The Re values achieved in the present study are within a range of $2.7-3.2 \times 10^{4}$. According to Zdravkovich ${ }^{16}$, this Reynolds number range falls in the so-called subcritical regime, characterized by the formation of laminar-turbulent transition eddies in the separated shear layers.

\section{Techniques of Data Analysis}

\subsection{Wavelet transformation}

In order to provide a quantitative description of the instantaneous flow behaviors inferred by the MEMS sensor signals, the technique of Wavelet transformation was employed. Wavelet transformation (WT) of a signal trace $u(t)$ with respect to a wavelet function $\psi(t)$ is defined by a convolution integral ${ }^{17}$ :

$$
W(a, b)=\frac{1}{\sqrt{a}} \int u(t) \psi^{*}\left(\frac{t-b}{a}\right) d t
$$

where $a$ and $b$ are called the scale and translation, respectively, and $\psi^{*}$ is the complex conjugate of $\psi$. Following our previous experiences ${ }^{13,14,18}$, a Morlet wavelet function was adopted in the present Wavelet transformation. Thus, the mathematical expression of $\psi$ is shown below.

$$
\psi(T)=e^{i k_{\psi} T} e^{-T^{2} / 2}
$$

where the parameter $k_{\psi}$ was taken to be 6.0 to satisfy the admissibility condition. ${ }^{19}$ With $\psi$ given in (2), one can see that the Wavelet transformation in (1) appears like a finite-time Fourier transformation.

In this study, computation of Wavelet transformation was carried out with the MATLAB software. According to Torrence and Compo ${ }^{20}$, the wavelet coefficient $W(a, b)$ in (1) can be analytically transformed into $W(f, t)$, where $f$ and $t$ denote the frequency and 
time, respectively, of the signals analyzed. As a result, $W(f, t)$ can be referred to as a time-frequency spectrum, which provides the spectral information of the signal at a localized time. In the present Wavelet transformation, the vortex shedding frequency, $f_{s}$, at a time instant was determined as the one where the local maximum wavelet coefficient was found, provided that it would fall within $\pm 20 \%$ of $F_{\mathrm{s}}$, the peak frequency found in the corresponding Fourier spectrum. It was verified that the time-average value of $f_{s}$ agreed very well with the corresponding value of $F_{\mathrm{s}}$.

Further, the phase $\theta$ of $f_{s}$ at a time $t$ can be defined below.

$$
\theta\left(f_{s}, t\right)=\tan ^{-1} \frac{I\left(W\left(f_{s}, t\right)\right)}{R\left(W\left(f_{s}, t\right)\right)}
$$

where $R$ and $I$ denote the real and imaginary parts of $W\left(f_{s}, t\right)$, respectively.

\subsection{Hilbert-Huang transformation}

The instantaneous vortex shedding frequency can also be reduced by the technique of Hilbert-Huang transformation (HHT). ${ }^{21}$ Mathematically, Hilbert Transformation of a real-value signal, $u(t)$, can be shown below. ${ }^{22}$

$$
u_{h}(t)=\mathrm{H} \quad[u(t)]=\int_{-\infty}^{\infty} \frac{u\left(t^{\prime}\right)}{\pi\left(t-t^{\prime}\right)} d t^{\prime}
$$

Let $z(t)=u(t)+i u_{h}(t)$, which is an analytic complex function. $z(t)$ can also be written as $z(t)=A(t) \cdot e^{i \theta(t)}$, where $A(t)$ is called the envelope of $u(t)$, and $\theta(t)$ is called the instantaneous phase of $u(t)$.

$$
\theta(t)=\tan ^{-1}\left[\frac{u_{h}(t)}{u(t)}\right]
$$

By differentiating $\theta(t)$ with respect to time, the instantaneous frequency, $f(t)$, of $u(t)$ can therefore be obtained.

$$
f(t)=\frac{1}{2 \pi} \frac{\mathrm{d} \theta(t)}{\mathrm{dt}}
$$

However, a priori of applying Hilbert transformation to a signal is that the signal should contain no DC component. ${ }^{21}$ Because of this consideration, Huang et al. ${ }^{21}$ proposed a procedure called the Empirical Mode Decomposition (EMD) to decompose a continuous signal into a set of mono-components. Thus, each Intrinsic Mode Function (IMF) resulted from the EMD procedure is ready for Hilbert transformation. One may refer to Huang et $a l^{21}$ for further details of this technique, which is relevant to analyzing the non-stationary, nonlinear data.

By applying HHT to a time trace of the MEMS sensor signals measured, the dominant IMF which contained the fluctuations associated with the vortex shedding frequency component could be identified and subsequently analyzed by Hilbert transformation.

\section{Results and Discussion}

\subsection{Comparison of the signals of the MEMS sensors and the hot-wires}

In order to verify the reliability of the measurement data obtained by self-made MEMS sensors, experiments were conducted in the wind tunnel shown in Fig. 3(b) for comparing the signals of the MEMS sensors and hot-wire probes positioned in the flow. Figure 5 shows a schematic drawing of four MEMS sensors on a circular cylinder model whose aspect ratio was 12.5 , and two hot-wire probes situated very close to the cylinder surface. Namely, the four MEMS sensors were aligned spanwisely on the cylinder surface at $\theta=75^{\circ}$, $\mathrm{z}= \pm 0.5 \mathrm{D}, \pm 1.5 \mathrm{D}$, named $\mathrm{S} 1$ to $\mathrm{S} 4$ from the left end of the cylinder. As learned from the previous study ${ }^{13}$, at this circumferential location where the sensors were located the boundary-layer flow on the circular cylinder would not be separated yet. Also shown in 
the figure are the two normal hot-wire probes a and $\mathrm{b}$, located at $(\mathrm{x}, \mathrm{y}, \mathrm{z})=(-0.0825 \mathrm{D}, 0.5 \mathrm{D}$, $\pm 1.5 \mathrm{D}$ ), very close to the MEMS sensors S1 and S4, respectively.

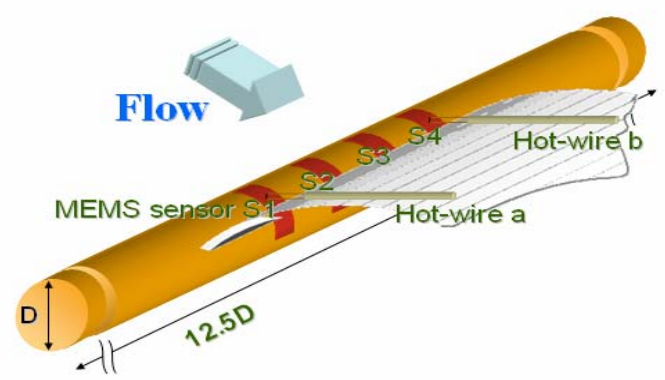

Figure 5 Four MEMS sensors spanwisely distributed on a circular cylinder with two hot-wire probes positioned very closed to the sensors S1 and S4, respectively.

The signal traces of the MEMS sensors, S1 and S4, and the two hot-wire probes obtained within a sampled period of 0.4 seconds, at $R e=3.2 \times 10^{4}$, are presented in Figure 6 for comparison. In Figure 6 (a), the signals traces of the MEMS sensors appear to be dominated by the fluctuations associated with the vortex shedding frequency component. Meanwhile, the amplitudes of the fluctuations show noticeable modulations at much lower frequencies. Similar characteristics can also be found in Fig. 6 (b) for the signal traces of the two hot-wire probes.

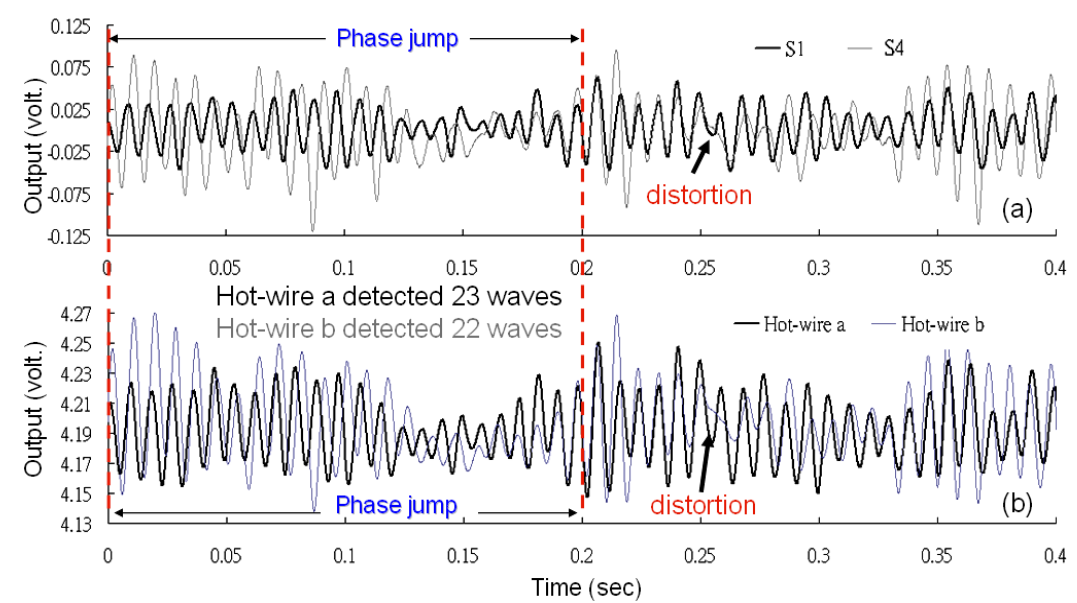

Figure 6 The signal traces of (a) the MEMS sensors at $\theta=75^{\circ}$ and (b) hot-wire probes at (x, y, $\mathrm{z})=(-0.0825 \mathrm{D}, 0.5 \mathrm{D}, \pm 1.5 \mathrm{D})$, for a time period of 0.4 seconds, at $\mathrm{Re}=3.2 \times 10^{4}$.

In fact, some interesting features can be noticed from Fig. 6 as one examines the signal traces in detail. Within the time period of $t=0-0.2$ seconds the signal trace of the MEMS sensor S1 or the hot-wire a contain 23 cycles of fluctuations, whereas the signal trace of S4 or the hot-wire b contain 22 cycles of fluctuations only. Moreover, in the neighborhood of $\mathrm{t}=0.25$ second, both the signal traces of the MEMS sensor $\mathrm{S} 4$ and the hot-wire probe $\mathrm{b}$ show that the fluctuations associated with the vortex shedding frequency component can not be clearly identified, unlike those of the signal trace of MEMS sensor S1 or the hot-wire a. Since the MEMS sensor S1 or the hot-wire a was situated 3 D away from the MEMS sensor $\mathrm{S} 4$ or the hot-wire b spanwisely, the spanwise inconsistency noticed clearly evidenced the presence of three-dimensionality of vortex shedding.

Further analysis of the signal traces shown in Fig. 6 was carried out with the techniques of WT and HHT described above. Figure 7 presents the results of these two approaches for discussion. First of all, either Fig. 7 (a) or (b) indicates that temporal variations of spanwise phase differences associated with the vortex shedding frequency regarding the instantaneous phase of S4 relative to that of S1 and the instantaneous phase of the hot-wire $\mathrm{b}$ relative to that of the hot-wire a are in good agreement. This implies that these MEMS sensors on the surface of the cylinder are able to sense the process of vortex shedding as the hot-wire probes in flow. Secondly, comparing Figs. 7 (a) and (b) reveal 
some interesting findings, which are worth mentioning here. As indicated in both plots, there is a dramatic increase of phase, $1.5 \pi$, occurred about $t=0.15$ second, then approaching to $2 \pi$ later on, which clearly indicates the occurrence of vortex dislocation. As pointed out earlier in Fig. 6, in the neighborhood of this time instant the fluctuating amplitudes of the signal traces are strongly modulated. Williamson ${ }^{8,9}$ also addressed that the phenomenon of vortex dislocation took place at the time when the vortex shedding signals appeared strongly modulated. On the other hand, a discrepancy is noted between the plots of Figs. 7 (a) and (b) in the neighborhood of $\mathrm{t}=0.25$ second, that in Fig. 7 (b) a phase jump is noted, whereas in Fig. 7 (a) no phase jump is seen. This enlightens the possibility of inconsistency between the results reduced by the two methods. Referring to the signal traces in Fig. 6, the event of phase jump revealed by the HHT analysis in Fig. 7 (b) is clearly due to the local distortion of the signals seen from the MEMS sensor S4 or the hot-wire b. Failure in identifying this particular event by the WT analysis in Fig. 7 (a) is attributed to that the vortex shedding frequency was not able to be clearly identified during the time period. Note that in the analysis of WT, the phase of the vortex shedding frequency component is reduced after the identification of the vortex shedding frequency, seen in (3). On the other hand, in the analysis of HHT, the phase of vortex shedding frequency component is obtained directly by Hilbert transformation, seen in (5).

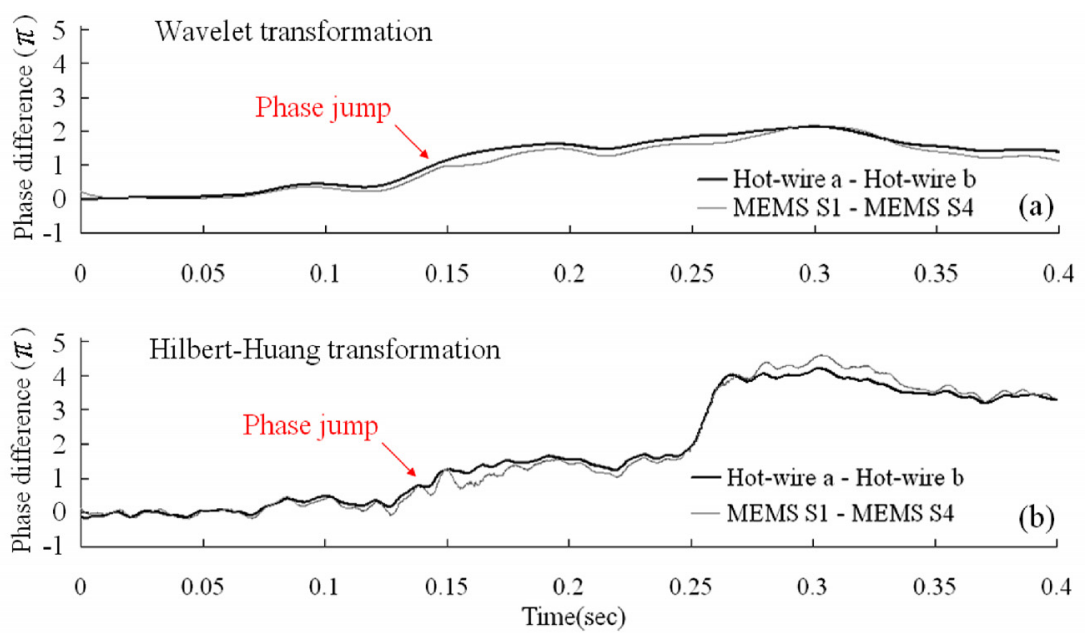

Figure 7 Temporal variations of spanwise phase difference concerning the vortex shedding frequency component, (a) the WT analysis and (b) the HHT analysis.

Figure 8 further compares the results of the instantaneous vortex shedding frequency, $f_{s}$, reduced by the two methods, based on the signal traces shown in Fig. 6. This comparison reveals that the HHT results in Fig. 8 (b) contain much more small-scale fluctuations compared to those of WT in Fig. 8 (a), although the mean values agree quite well. In fact, the mean value is also coincided with that reduced from Fourier spectral analysis, $F_{s}=115 \mathrm{~Hz}$. The small-scale fluctuations seen in Fig. 8 (b) could be due to the numerical differentiation shown in (6) mainly. Nevertheless, large amplitude fluctuations seen around $\mathrm{t}=0.15$ and 0.25 seconds are clearly due to the occurrences of vortex dislocation. Actually, in identifying the vortex shedding frequencies at the two time instants, both analysis techniques show their deficiencies, because the fluctuations associated with the vortex shedding frequency component can not be clearly seen in the signals. Consequently, in the HHT analysis, the resultant values of vortex shedding frequency showed pronounced variations with respect time; in the WT analysis, extra efforts were needed in order to identify the $f_{s}$ values.

Further experiments were made to examine the correlations of the $f_{s}$ values reduced from the signals of the MEMS sensors and the hot-wires introduced in the flow. Table 1 shows the correlation coefficients $(\rho)$ associated with the $f_{\mathrm{s}}$ values reduced from the signals of S1 - S4, and the two hot-wire probes positioned in the near wake region. The correlation analysis was made based on the signals sampled for 60 seconds. Note that the $f_{\mathrm{s}}$ values were obtained by the WT method, because the $f_{\mathrm{s}}$ values reduced by the HHT method 
contained more small-scale fluctuations as mentioned. In Case 1 for which the hot-wire probes a and b were situated very close to the MEMS sensors 1 and 4, respectively, either the correlation coefficient of S1 and the hot-wire a or that of S4 and the hot-wire b appears very close to 1 . On the other hand, the values associated with spanwise correlations show significantly less, which indicate the significance of three-dimensionality of vortex shedding. In Cases 2 and 3 where the hot-wire probes were located at $\mathrm{x}=1 \mathrm{D}$ and $2 \mathrm{D}$, respectively, the coefficient values of S1 and the hot-wire a, and those of S4 and the hot-wire $b$ are higher than 0.7 , signifying that the characteristics of vortex shedding flow structures persist quite well downstream. Thus, one can argue that a vortex dislocation event observed near the surface of the cylinder shall carry on further downstream in the wake flow. Alternately, one could also comment that the phenomenon of vortex dislocation seen at the present Reynolds numbers is global in the sense that it can be detected as far as upstream at the cylinder and downstream in the near wake.
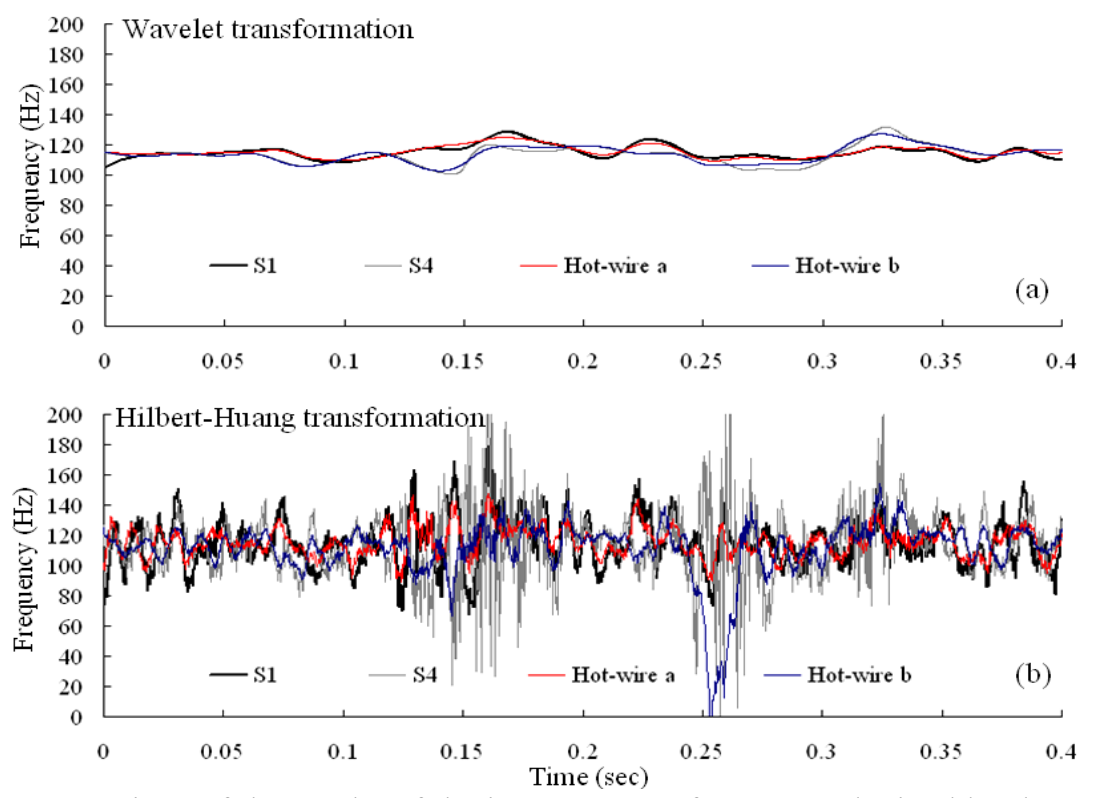

Figure 8 Comparison of the results of the instantaneous frequency obtained by the WT and HHT analyses for the signal traces shown in Figure 6.

Table 1 Correlation coefficients between the instantaneous vortex shedding frequency values reduced by MEMS sensors $\mathrm{S} 1$ and $\mathrm{S} 4$ on the cylinder surface and hot-wire probes at $(\mathrm{x}, \mathrm{y}, \mathrm{z})=(-0.0825 \mathrm{D}, 0.5 \mathrm{D}, \pm 1.5 \mathrm{D}),(1 \mathrm{D}, 1 \mathrm{D}, \pm 1.5 \mathrm{D})$ and $(\mathrm{x}, \mathrm{y}, \mathrm{z})=(2 \mathrm{D}, 1.7 \mathrm{D}, \pm 1.5 \mathrm{D})$.

\begin{tabular}{|c|c|c|c|c|c|c|}
\hline Case 1 & \multicolumn{6}{|c|}{ Hot-mire probes at $(\mathrm{z}, \mathrm{y}, \mathrm{z})=(-0.0825 \mathrm{D}, 0.5 \mathrm{D}, \pm 1.5 \mathrm{D})$} \\
\hline Sensors & S1-\$2 & $\mathrm{S} 1 \$ 3$ & \$1-\$4 & S1-HWa & HWa-HWb & HWb-S4 \\
\hline Con. $(\rho)$ & 0.554 & 0298 & 0.202 & 0.997 & 0278 & 0.998 \\
\hline Case 2 & \multicolumn{6}{|c|}{ Hot-mire probes at $(x, y, z)=(1 D, 1 D, \pm 1.5 D)$} \\
\hline Sensors & S1-S2 & S1-33 & S1-S4 & S1-HWa & HWa-HWb & $\mathrm{HWb}-\mathrm{S4}$ \\
\hline Con. $(\rho)$ & 0.547 & 0.307 & 0.186 & 0.739 & 0294 & 0.759 \\
\hline Case 3 & \multicolumn{6}{|c|}{ Hot-rire probes at $(\mathrm{z}, \mathrm{y}, \mathrm{z})=(2 \mathrm{D}, 1.7 \mathrm{D}, \pm 1.5 \mathrm{D})$} \\
\hline Sensors & S1-\$2 & S1-\$3 & \$1-\$4 & S1-HWa & HWa-HWb & HWb-S4 \\
\hline Corr. $(\rho)$ & 0.490 & 0278 & 0.209 & 0.708 & 0.316 & 0.716 \\
\hline
\end{tabular}

\subsection{Wavelet analysis of the signals obtained by MEMS sensors in spanwise array}

The results presented in this section were obtained from the experiments carried out in the closed-return low-speed wind tunnel shown in Fig. 3 (a). Referring to Fig. 4, an array of MEMS sensors were situated on a circular cylinder, whose aspect ratio was 4.75 . The sensors were equally spaced at $1.6 \mathrm{~mm}$ between two neighboring ones. In the experiment, 14 sensors were selected for measurement, named S1 to S14 indicated in Figure 4(b). These sensors were positioned symmetrically with respect to $\mathrm{z}=0$ along the spanwise 
direction. Specifically, they were located at $\mathrm{z}= \pm 0.1 \mathrm{D}, \pm 0.3 \mathrm{D}, \pm 0.7 \mathrm{D}, \pm 1.1 \mathrm{D}, \pm 1.5 \mathrm{D}, \pm 1.9 \mathrm{D}$ and $\pm 2.3 \mathrm{D}$, respectively. Since the thickness of the boundary layer on the sidewall measured was about $20 \mathrm{~mm}$, equivalent to $0.625 \mathrm{D}$, the sensors S1 and S14 were actually immersed in the boundary layers on the two side walls, respectively. During the experiment, the sensors were positioned at $\theta=75^{\circ}$ for sensing unsteady flow motions in the boundary layer developed on the cylinder prior to separation.

Figure 9 presents the MEMS sensor signals of S1 - S7 obtained at $\mathrm{Re}=2.7 \times 10^{4}$ over a time length of 1 second for discussion. As noted, while the fluctuations in the signal traces appear to be dominated by the vortex shedding frequency component, they reveal a feature in common that the fluctuating amplitudes are strongly modulated. Specifically, the fluctuating amplitudes of the sensors S2-S7 are strongly modulated in the neighborhood of $\mathrm{t}=0.15$ and 0.5 seconds, where $\mathrm{t}=0$ was arbitrarily chosen.

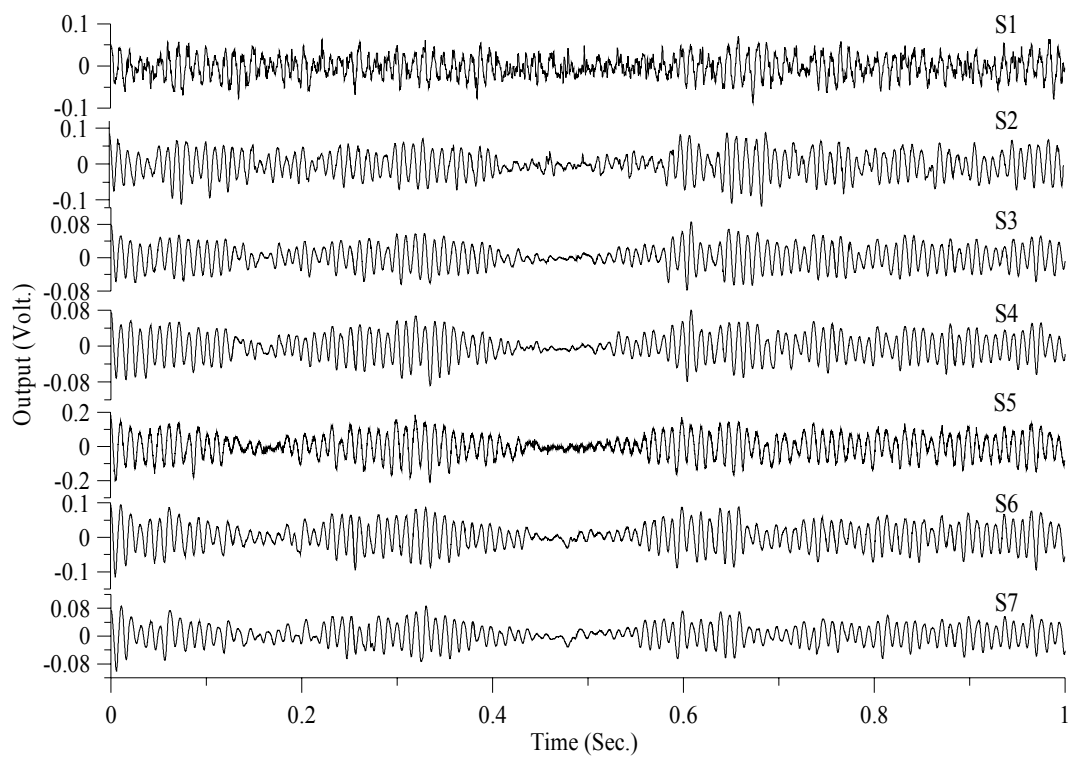

Figure 9 MEMS sensor signals of S1 - S7 obtained at $\mathrm{Re}=2.7 \times 10^{4}$ over a time length of one second.
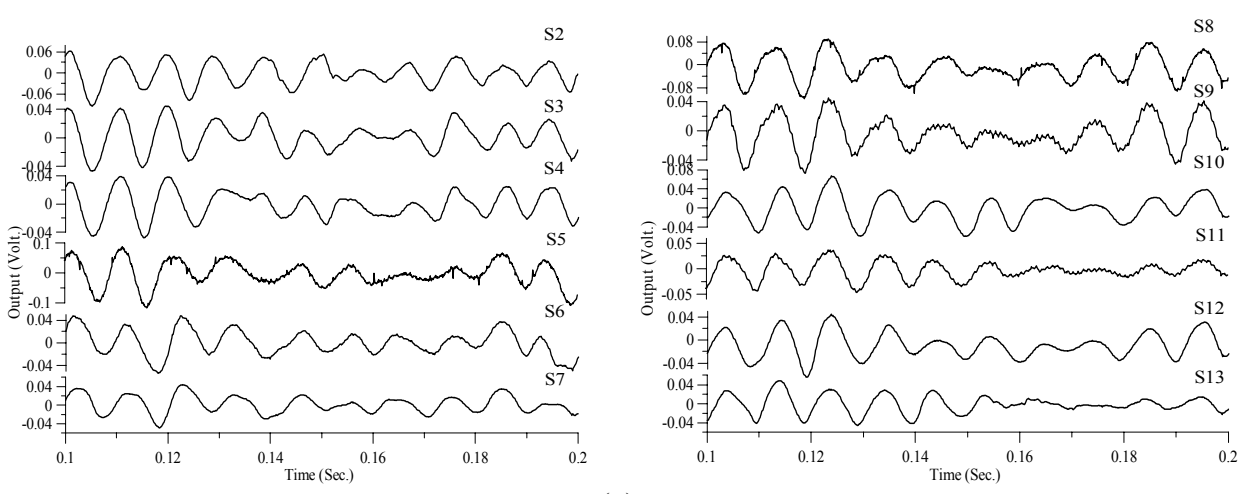

(a) 


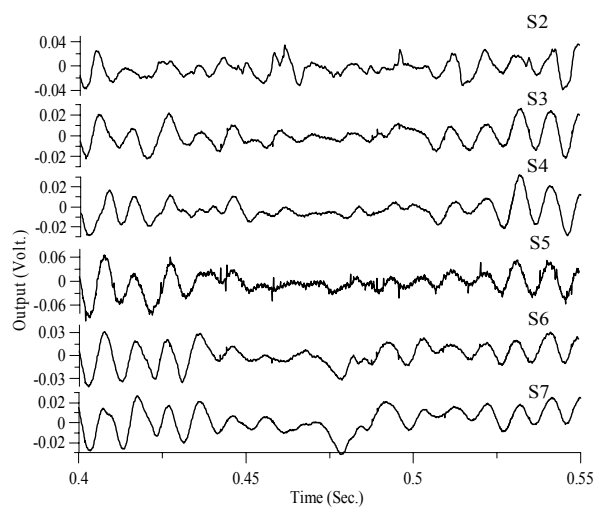

S3 S5 $\sqrt{1}$

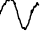

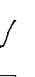

(b)

Figure 10 Signal traces of the sensors $\mathrm{S} 2$ to $\mathrm{S} 13$ obtained at $\mathrm{Re}=2.7 \times 10^{4}$ within the time periods of (a) $t=0.1-0.2 \mathrm{sec}$ and (b) $t=0.4-0.55 \mathrm{sec}$.

To examine the three-dimensionality of vortex shedding in the neighborhood of these instants, Figs. 10 (a) and (b) further present the signal traces of the sensors S2 to S13 within the time periods of $\mathrm{t}=0.1-0.2$ seconds and $\mathrm{t}=0.4-0.55$ seconds, respectively, in detail. As seen, not only the phase of vortex shedding frequency component varies widely among the sensors, but also at some instants the fluctuations associated with the vortex shedding frequency component can not be clearly identified. Above observations imply that within these time periods, the process of vortex shedding was unlikely in phase along the span, and even could be diminished at some time instants.

The WT analysis was performed to extract the quantitative information contained in the MEMS sensor signals measured, particularly on the three-dimensional characteristics of vortex shedding. Since the three-dimensionality of vortex shedding infers non-zero phase differences of the vortex shedding along the span of the cylinder, it is of interest here to examine in detail the phase relations of vortex shedding at the spanwise locations of the MEMS sensors. Figure 11 presents the temporal variations of spanwise phase differences at $f_{\mathrm{s}}$ in reference to the phase of $\mathrm{S} 2$, for a time length of 1 second, at $\mathrm{Re}=2.7 \times 10^{4}$. In this figure, within $\mathrm{t}=0.1-0.2$ seconds large phase variations can be identified among the sensors of S5 - S13; the other event of large phase variations can be identified among the sensors of $\mathrm{S} 3-\mathrm{S} 13$, around $\mathrm{t}=0.42-0.52$ seconds. These observations infer that within $\mathrm{t}=0.1-0.2$ seconds strong three-dimensionality of vortex shedding was occurred in the region over the sensors S4 and S5, which led to a result of spanwise phase jump. As seen in the figure, the phase difference value is amounted to $2 \pi$, inferring a situation of vortex dislocation. Further, within $\mathrm{t}=0.42-0.52$ seconds the results shown imply that strong three-dimensionality of vortex shedding actually prevailed over the entire span of the cylinder. Referring to Fig. 10 (b), within this time period all the signals traces of the sensors S2 - S13 were distorted seriously. As a result, phase jumps of $2 \mathrm{~N} \pi(\mathrm{N}$ denoting integer) are remarkably seen in the curves in Fig. 11. 


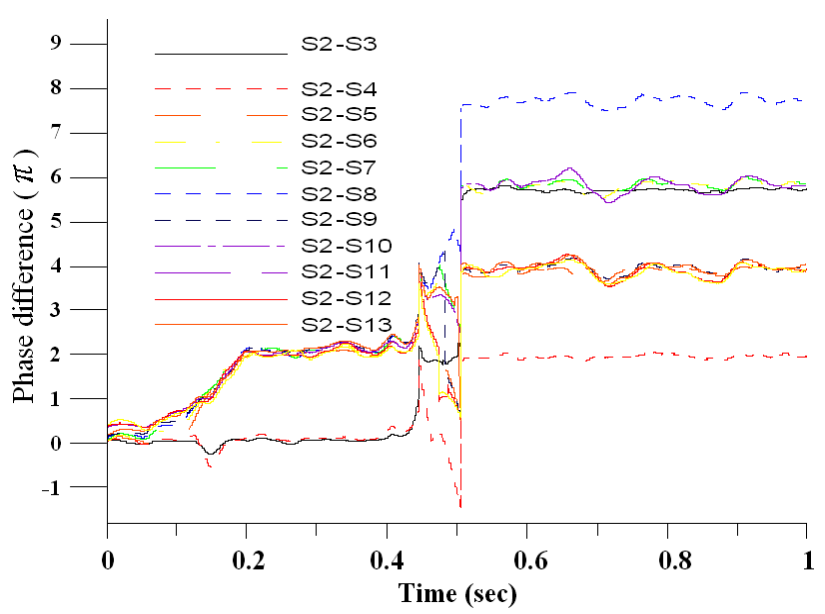

Figure 11 Temporal variations of the spanwise phase differences at $f_{s}$ in reference to the phase at the location of S2, for a time length of 1 second, at $\mathrm{Re}=2.7 \times 10^{4}$.

As a counterpart of Fig. 11, Fig. 12(a) compares the temporal variations of $f_{s}$ reduced from the signals of the sensors S2 - S13. In this figure, the three-dimensional events can be realized as when pronounced variations take place among the $f_{s}$ curves of S2 - S13. These appearances further suggest that the root-mean-square $(\mathrm{rms})$ value of $f_{s}$ reduced from the sensors S2 - S13 at each time instant be a good indication of the three-dimensionality of vortex shedding. As a result, Fig. 12(b) presents temporal variations of the rms value of $f_{s}$ mentioned, which has been normalized by $F_{s}$. It is clearly seen in the figure that within the two time intervals mentioned above, the rms values of $f_{s}$ appear much greater than those at other time instants. In viewing of this, a threshold value, $5 \%$ of $F_{s}$, is introduced in the figure to single out the time periods featuring strong three dimensionality of vortex shedding. Further, applying this discriminating criterion to the signals sampled over a time period of 180 seconds found that about $10 \%$ of time three-dimensionality of vortex shedding appeared to be pronounced. ${ }^{15}$

The three-dimensional events can also be realized from a space-time map shown in Fig. 13, which indicate the degree of the three-dimensionality of vortex shedding with respect to time and the spanwise location measured. In the figure, each grey level represents an rms value of $f_{s}$, normalized by $F_{s}$, reduced from the signals of a sensor over a time length of $50 \mathrm{msec}$. It should be mentioned that in this figure, the results were reduced based on the signal traces of the 14 sensors within a time period of $0-1$ seconds, seven of which are shown in Fig. 9. As seen in the figure, in the neighborhoods of $\mathrm{t}=0.15$ and 0.45 seconds, the grey levels appear much brighter, indicating higher rms values. Specifically, at $\mathrm{t}=0.15$ second, in the region of $\mathrm{z}=1.1-0.7 \mathrm{D}$, corresponding to the sensors $\mathrm{S} 4$ and $\mathrm{S} 5$, the rms values of $f_{s}$ appear significantly larger than those at other spanwise positions. At $\mathrm{t}=0.45$ second, high grey level prevailing over the entire span, while the maximum value takes place in the region of $\mathrm{z}=1.9 \mathrm{D}$ to $0.7 \mathrm{D}$. Therefore, at this moment, strong three-dimensionality of vortex shedding appear to take place over the entire span of circular cylinder. The arrowheads in Figure 13 further indicate the time instants and spanwise locations where the events of phase jump took place, according to the results of Wavelet analysis reported earlier. 


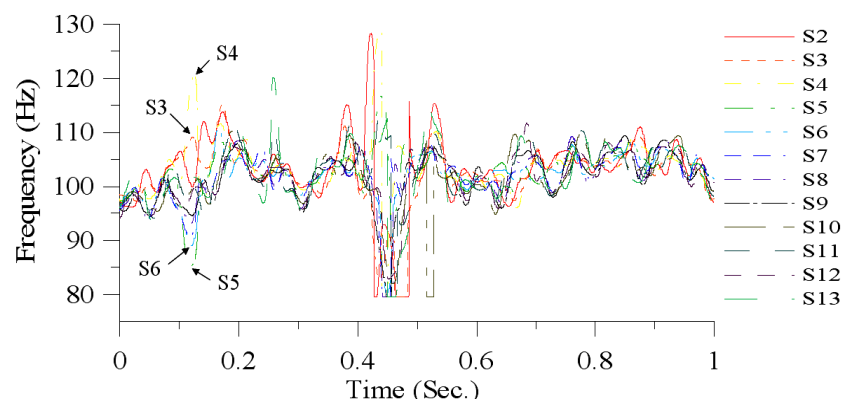

(a)

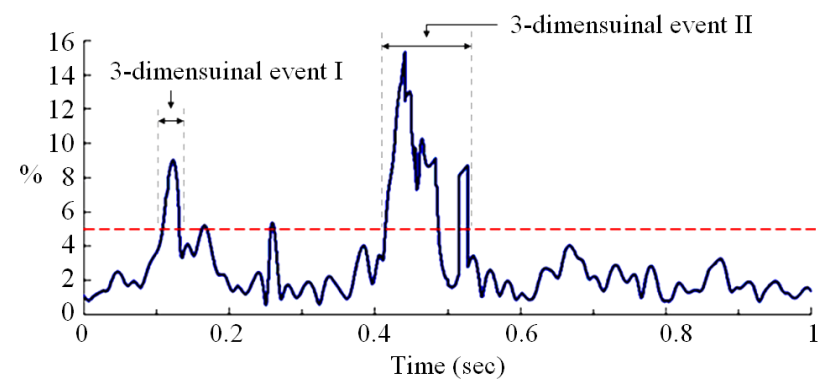

(b)

Figure 12 (a) Temporal variations of $f_{s}$ of S2 - S13 for a time length of 1 second, at $\mathrm{Re}=2.7 \times 10^{4}$. (b) Temporal variations of the standard deviation of the $f_{s}$ values of S2-S13.

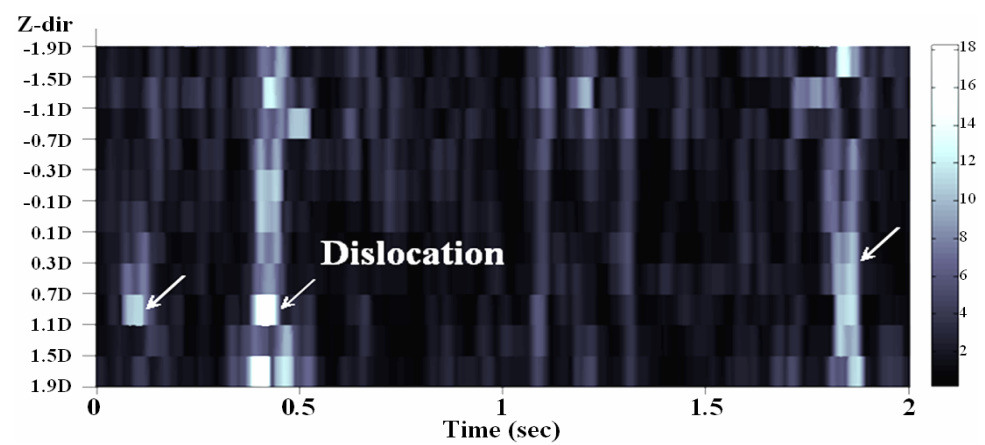

Figure 13 Space-time map concerning the occurrences of vortex dislocation for flow over the circular cylinder at $\mathrm{Re}=2.7 \times 10^{4}$.

\subsection{D-FFT analysis of the signals MEMS sensors in spanwise array}

An experiment was conducted in the low speed wind tunnel shown in Fig. 3 (a) to examine the characteristic spanwise wave numbers of vortex shedding. This was made with 16 MEMS sensors positioned equidistantly on the surface of the circular cylinder over a spanwise region of $3 \mathrm{D}$, at $\theta=75^{\circ}$. The signal traces of the multiple sensors were subsequently analyzed with a 2D-FFT algorithm.

Figure 14 presents the 2D-FFT spectral results reduced over a time length of 60 seconds, at $\mathrm{Re}=2.7 \times 10^{4}$. Remarkable features noted from the figure can be described below. Firstly, it is found that the characteristic vortex shedding frequency appears to be varied over a band of $85-110 \mathrm{~Hz}$, and most likely located at about $100 \mathrm{~Hz}$. Secondly, along the axis of spanwise wave number it is found that most of fluctuating energy falls in a range of the wave numbers -2 to 2 . Note that the wave number zero represents the mode of two-dimensional vortex shedding, corresponding to the component of in-phase fluctuations of the 16 MEMS sensors over the span of 3 D. Conversely, the fluctuations associated with other wave numbers imply the three-dimensional motions. 


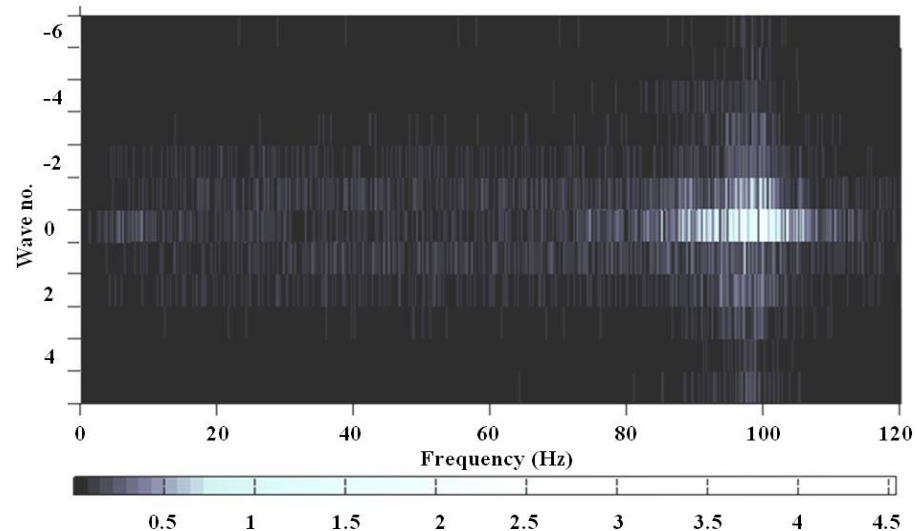

Figure 14 2D-FFT spectrum reduced from the signals of 16 MEMS sensors at $\mathrm{Re}=2.7 \times 10^{4}$.

In order to examine the events of pronounced three-dimensional vortex shedding, the 2DFFT analysis was performed for some selected segments of raw signals. Figure 15 compare two cases of which the vortex shedding processes were sampled over 0.1 seconds in time. The left hand side in Fig. 15 (a) represents a case of vortex shedding being two-dimensional like, for which all the signal traces of the 16 MEMS sensors appear almost coincided. The corresponding 2D-FFT spectrum in Fig. 15 (b) indicates that the peak value is clearly located at zero wave number and $100 \mathrm{~Hz}$ in frequency. On the other hand, the right hand side in Fig. 15 (a) represents a case of which the vortex shedding process behaves strongly three-dimensional, i. e., the signal traces of the 16 MEMS sensors show widely different in temporal variations. The corresponding 2D-FFT spectrum in Fig. 15 (b) indicates that the wave packets appear in widespread distribution. Along the axis of spanwise wave number, one can read that the peak value falls in the neighborhood of -1 , indicated by the arrowhead. This implies that during the time period sampled, there exists a skewed wavy pattern of vortex shedding along the spanwise direction, in the region covered by the 16 MEMS sensors.
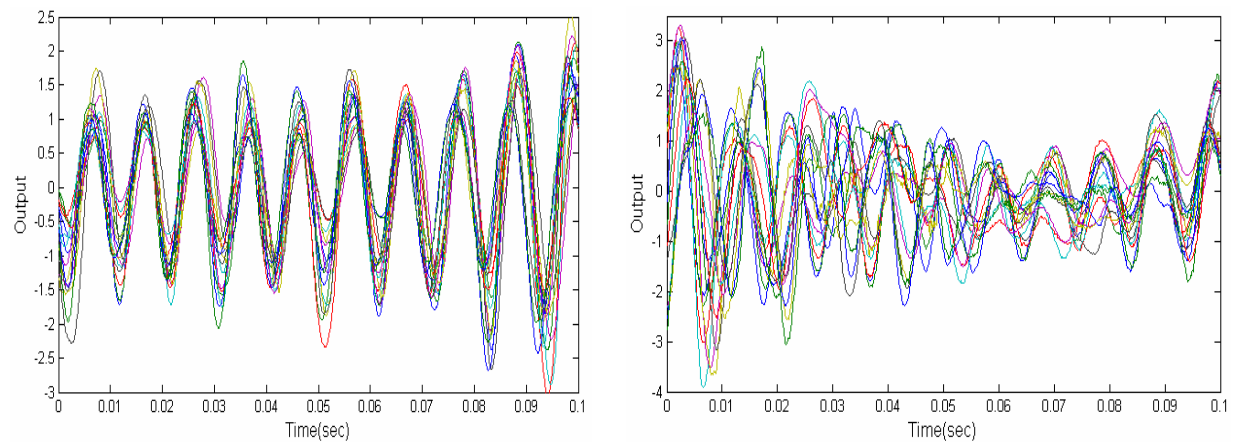

(a)
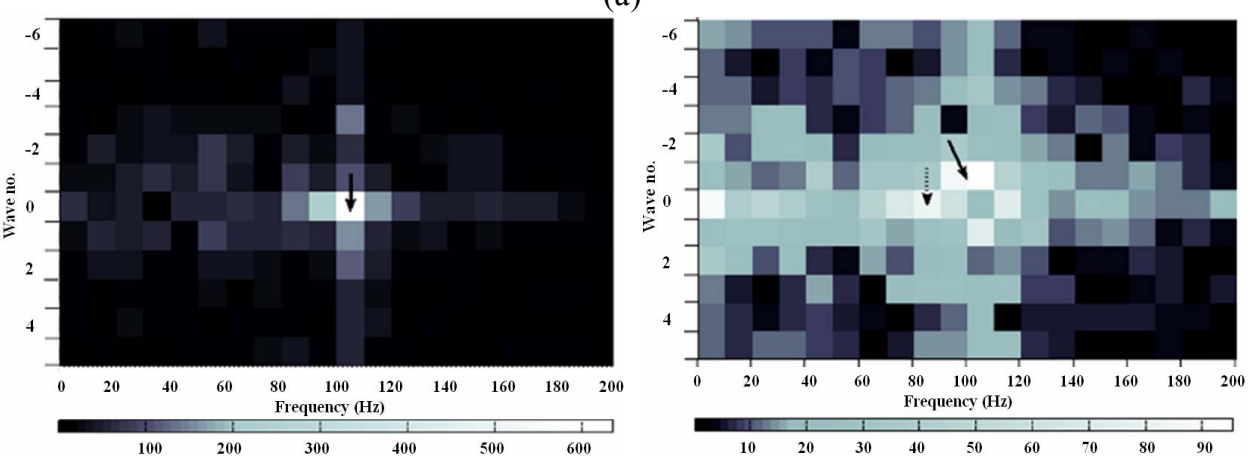

(b)

Figure 15 Results reduced from the 16 MEMS sensors on the circular cylinder at $\mathrm{Re}=2.7 \times 10^{4}$, (a) the signal traces associated with the cases of the two-dimensional like vortex shedding (left) and the three-dimensional vortex shedding(right); (b) the corresponding short-time 2D-FFT spectra. 


\section{Concluding Remarks}

Substantial understandings have been obtained in this study on the unsteady, three-dimensional behaviors of vortex shedding from circular cylinder at Reynolds numbers of $10^{4}$. Major findings can be summarized below.

Firstly, the Wavelet and Hilbert-Huang transformations were successfully applied to analyze the signals of multiple MEMS sensors situated spanwisely on the cylinder. Strongly three-dimensional events of vortex shedding were recognized as associated with the occurrences of vortex dislocation. These events would take place randomly in time and in a limited spanwise region or over the entire cylinder. This finding has a further implication that for the present Reynolds numbers studied the phenomenon of vortex dislocation is actually initiated from the proximity of the bluff body, subsequently carrying further downstream in wake flow. According to the statistics reduced from the signals measured, one can say that about $10 \%$ of time the process of vortex shedding behaved strongly three-dimensional.

Secondly, the results of 2D-FFT analysis on the MEMS signals obtained in a spanwise extent of $3 \mathrm{D}$ show that the dominant spanwise wave numbers of vortex shedding largely falls in a range between the wave numbers of -2 to 2 . This implies that the characteristic wave length of three-dimensional vortex shedding is in the order of $1 \mathrm{D}$, which is noted in good agreement with the finding reported by Szepessy. ${ }^{23}$

\section{Acknowledgment}

The authors would like to acknowledge the funding support of National Science Council, Taiwan, ROC, on this work, under the contract numbers of 95-2221-E-006-243.

\section{References}

[1] Roshko, A., "On the Development of Turbulent Wakes from Vortex Streets," NACA Report 1191, 1954.

[2] Roshko, A., "Perspectives on Bluff Body Aerodynamics," Journal of Wind Engineering and Industrial Aerodynamics, Vol. 49, 1993, pp. 79-100.

[3] Norberg, C., "Fluctuating Lift on a Circular Cylinder: Review and New Measurements," Journal of Fluids and Structures, Vol. 17, 2003, pp. 57-96.

[4] Norberg, C., "An Experimental Investigation of the Flow Around a Circular Cylinder: Influence of Aspect Ratio," Journal of Fluid Mechanics, Vol. 258, 1994, pp. 287-316.

[5] Szepessy, S. and Bearman, P. W., "Aspect Ratio and End Plate Effects on Vortex Shedding from a Circular Cylinder," Journal of Fluid Mechanics, Vol. 234, 1992, pp. 191-217.

[6] Braza, M., Faghani, D., and Persillon, H., "Successive Stages and the Role of Natural Vortex Dislocations in Three-Dimensional Wake Transition," Journal of Fluid Mechanics, Vol. 439, 2001, pp. 1-41.

[7] Henderson, R. D., "Nonlinear Dynamics and Pattern Formation in Turbulent Wake Transition," Journal of Fluid Mechanics, Vol. 352, 1997, pp. 65-112.

[8] Williamson, C. H. K., "The Natural and Forced Formation of Spot-Like Vortex Dislocations in the Transition of a Wake", Journal of Fluid Mechanics, vol. 243, 1992, pp. 393- 441.

[9] Williamson, C. H. K., "Advances in our Understanding of Vortex Dynamics in Bluff Body Wakes", Journal of Wind Engineering and Industrial Aerodynamics, Vol.69-71, 1997, pp. 3-32.

[10] Yang, P. M., Mansy, H. and Williams, D. R., "Oblique and Parallel Wave Interaction in 
the Near Wake of a Circular Cylinder”, Phys. Fluids A, vol. 5, 1993, pp. 1657-1661.

[11] Blackburn, H. M. and Melbourne, W. H., "The Effect of Free-Stream Turbulence on Sectional Lift Forces on a Circular Cylinder", Journal of Fluid Mechanics, vol. 306, 1996, pp. 267-292.

[12] Eisenlohr, H., Eckelmann, H., "Vortex Splitting and its Consequences in the Vortex Street Wake of Cylinders at Low Reynolds Number", Phys. Fluids A, no.1, 1989, pp. 189-192.

[13] Miau, J. J., Tu, J. K., Chou, J. H., and Lee, G. B., "Sensing Flow Separation on a Circular Cylinder by MEMS Thermal-Film Sensors," AIAA Journal, Vol. 44, No. 10, 2006, pp. 2224-2230.

[14] Tu, J. K., Miau, J. J., Wang, Y. J., Lee, G. B., and Lin, C., "Studying Three-Dimensionality of Vortex Shedding at Reynolds Numbers of $10^{4}$ with MEMS Sensors," AIAA Paper 2006-1413, presented at 44th AIAA Aerospace Sciences \& Exhibit, Reno, Nevada, 9-12 January 2006.

[15] Tu, J. K., "Investigation of Unsteady, Three-Dimensional Characteristics of the Vortex Shedding behind Bluff Bodies at Sub-Critical Reynolds Numbers" Ph.D. thesis, Department of Aeronautics and Astronautics, National Cheng Kung University, Tainan, Taiwan, R. O. C. 2007.

[16] Zdravkovich, M. M., Flow Around Circular Cylinder, Vol. 1: Fundamentals, Oxford University Press, 1997.

[17] Grossman, A. and Morlet, J., "Decomposition of Hardy Functions into Square Integrable Wavelets of Constant Shape," SIAM J. Math. Anal., Vol.15, 1984, pp. 723-736.

[18] Wu, S. J., Miau, J. J., Hu, C. C. and Chou, J. H. “On Low-Frequency Modulations and Three-Dimensionality in Vortex Shedding Behind a Normal Plate," Journal of Fluid Mechanics, Vol. 526, 2005, pp. 117-146.

[19] Farge, M. "Wavelet Transforms and Their Applications to Turbulence," Annu. Rev. Fluid Mech., Vol. 24, 1992, pp. 395-457.

[20] Torrence, C. and Compo, G. P. "A Practical Guide to Wavelet Analysis," Bull. Am. Meteorol. Soc., Vol. 79, 1998, pp. 61-78.

[21] Huang, N. E., Shen, Z., Long, S. R., Wu, M. C., Shih, H. H., Zheng, Q., Yen, N. C., Tung, C. C., and Liu, H. H., "The Empirical Mode Decomposition and the Hilbert Spectrum of Nonlinear and Non-Stationary Time Series Analysis," Proc. R. Soc. Lond., Vol. A454, 1998, pp. 903-995.

[22] Bendat, J. S., and Piersol, A. G., Random Data Analysis and Measurement Procedures. John Wiley \& Sons, Second Edition, 1991.

[23] Szepessy, S., 1994 "On the Spanwise Correlation of Vortex Shedding from a Circular Cylinder at High Subcritical Reynolds Number,” Phys. Fluids, Vol.6, 1994, pp. 24062416. 